\title{
NETWORK-BASED DESIGN OF HETEROGENEOUS MODULAR MOBILE ROBOTIC SYSTEMS
}

\author{
Victor P. Andreev \& Yury V. Poduraev
}
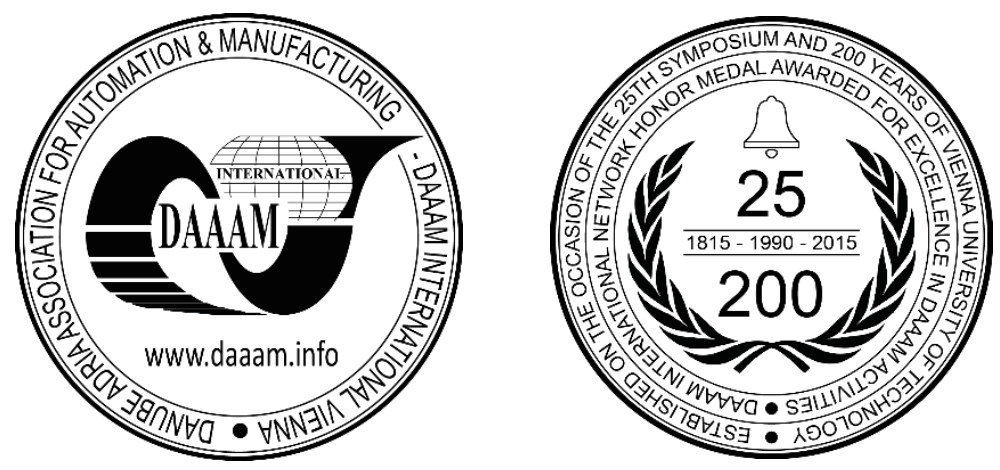

This Publication has to be referred as: Andreev, V[ictor] \& Poduraev, Y[uriy] (2016). Network-Based Design of Heterogeneous Modular Mobile Robotic Systems, Proceedings of the 27th DAAAM International Symposium, pp.00040009, B. Katalinic (Ed.), Published by DAAAM International, ISBN 978-3-902734-08-2, ISSN 1726-9679, Vienna, Austria

DOI: $10.2507 / 27$ th.daaam.proceedings.001

\begin{abstract}
The present work deals with a new approach to the construction of hardware and software part of the heterogeneous modular mobile robots. The new approach is to use a network principle for constructing information-measuring and control system of modular mobile robot. The paper discusses a functional-modular principle of construction of a heterogeneous mobile robot. Our concept is based on the functional completeness of each module, which is an electronic and/or mechatronic device with its own control system. The inclusion of any mechatronic devices in the network structure information measurement and control system of the mobile robot is performed via special software -driver.
\end{abstract}

Keywords: mobile robot, heterogeneous modular robot; local area network; information-measuring and control system

\section{Introduction}

Robotic systems are widely used in industry, medicine, and everyday life, especially in solving the problems related with emergency situations and defence missions. At present, the application of these systems usually depends on their structure, which leads to a large variety of design and engineering solutions. The main actuator, information and control components of these robotic systems are normally designed to be used in a particular system alone. The electronic and mechanical units of these systems cannot be structurally and functionally separated from their general design. Their software is unique and typically runs on a single powerful computer. All these factors make it difficult or even preclude the use these units in other similar systems. The fact that these robotic systems are expensive restricts the extent of their application.

The modular principle of robotic systems construction makes it possible to substantially scale down the variety of technical solutions, thus reducing the cost of final devices through the unification of hardware and relevant software. The modular architecture has long been used in machine-tool industry and makes it possible to develop flexible and faulttolerant systems with small mass-dimensional characteristics and low cost of manufacture. Therefore, the use of the modular principle in the design of mobile robotic systems it seems reasonable. In recent years began to appear publications which present the results of current scientific studies on the modular mobile robotics. 


\section{Modular robotics}

The modular principle of robotic systems design was proposed in the Russian State Scientific Center for Robotics and Technical Cybernetics (RTC, St. Petersburg, Russia) and first implemented in the Soviet Union as early as in the 1980s [1]. That time was characterized by the serial production of standard robotic components, such as electromechanical modules, control modules, and modular software systems. The second stage of progress in modular robotics can be attributed to the 1990s, when the accident at the Chernobyl nuclear power plant had occurred. By that time, the Scientific Center for Robotics and Technical Cybernetics prepared a large number of projects for different modules. Thanks to this, a total of 15 mobile robots designed for various purposes were manufactured and put into operation within a couple of months. These robots operated in the disaster area to survey the local radiation situation and clean the buildings and places from radioactive waste.

The experience of operation of these robotic systems resulted in a new research direction in modular robotics, the development of reconfigurable modular robots, i.e., robots with a variable structure. The maximum uncertainty in the conditions of future activities in case of emergency situations, accidents and active opposition makes it impossible to predetermine the composition and configuration of desired robotics. The use of reconfigurable modular robotics allows the robots to be assembled directly on site and adjust their structure in the course of operation.

The modular principle of robotic systems construction is a new research line with its main solutions having been implemented in the CEBOT robot [2] in 1987. The modular robots consist of individual components or nodes (modules), which are combined together to form an integral and independent robotic system. Each module can contain electronic, sensor, and/or control devices and actuators.

By their construction type, the modular robots can be divided into homogeneous and heterogeneous ones. In homogeneous robots, all modules are identical to each other and are separate functional units. These devices can change their configuration by restructuring their components. In heterogeneous robots, each module implements a separate function, which makes it possible to change the robot configuration by choosing an appropriate set of modules in line with the intended use of the robot.

By the method of their structure formation, the modular robots can be reconfigurable and self-reconfigurable. A considerable amount of well-known products in this field addresses self-reconfigurable homogeneous robots, such M-TRAN, ATRON, and SuperBot [3 - 5]. It is believed that in the long-term perspective the self-reconfigurable robots must be heterogeneous because the each module in homogeneous robots has to be additionally supplied with all the necessary components (sensors and/or actuators), which highly complicates the system [6]. Heterogeneous robots are more versatile and flexible [7 - 10] but usually not self-reconfigurable.

\section{Modern products of heterogeneous modular robots}

Thor (Fig. 1a) [7]. The concept of this robot is partially based on its "ancestor" - the Odin heterogeneous robot [8], the modules of which were morphologically homogeneous. A distinctive feature of Thor is that it is based on the submodular principle of the construction of functional units (a total of eight modules); each module executes only one simple function. In other words, certain function of a single unit of the robot must be divided between several simpler modules. For example, the gripping module (which is composed of the gripper itself, actuator, and batteries) is proposed to be replaced by sub functional modules: actuator, batteries, and a large set of gripping mechanisms, joints and wheels. The main advantage is that the individual submodules can be easily constructed, which allows other submodules to be quickly produced. More expensive submodules of the motor-module type are used only where required. Using different combinations of modules, one can construct mobile robots of different purposes.
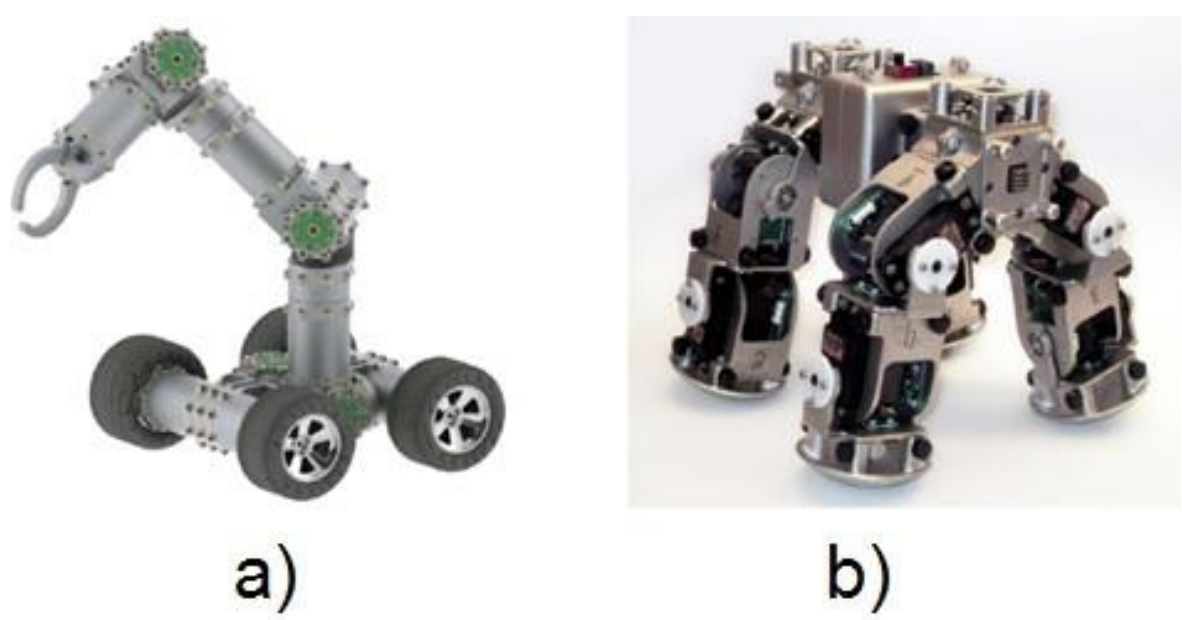

Fig. 1. Computer model of Thor (a); the robot for operations in space (b) 
The morphological heterogeneity and subfunctional modularity simplify the robot structure and increase the flexibility of its construction. However, if the modules become extremely simplified, the labour intensity in the construction assembly increases and the corresponding software becomes more complex as an element of the control system.

Robot for operations in space (Fig. 1b) [9]. Originally, the system involved three types of modules: module-actuator, node module, and communication and control module. The module-actuator is a module with servo-actuator and articulated coupling. The node module is a structure with five pin platforms allowing for different angles of coupling between the modules (including $90^{\circ}$ and $120^{\circ}$ ). Also, the node module provides power transmission and data exchange between coupled modules. The communication and control module allows the robot to operate autonomously or by wire and can be equipped with batteries. Then, "foot"-modules equipped with tactile sensors were developed. In addition, a new module (digital TV-camera) was introduced that can transmit images through a wireless channel to the host computer; this avoids the need for high capacity of the inter-module interface.

The modules communicate with each other and with one or more upper-level computers by using a special-purpose peering network. Any module can send data packets to another module, which ensures a distributed control. The resulting modules were tested in different configurations: a classic "snake-like" manipulator, a robot with multiple manipulators, and a walking robot with three or four walking drivers.

Due to the simple structure of modules, which is focused on rapid production, the use of new functional modules is simple and inexpensive. However, the use of a peer-to-peer network operating on the basis of the equality principle seems to be unjustified because the modules have different functional purposes and therefore are essentially unequal. In this case, the modules can only serve as a source of information (sensors) and/or receivers of simple instructions; the modules have no individual control system.

SMART [10]. This is a reconfigurable heterogeneous modular robotic system consisting of a set of interchangeable modules. The main purpose of this robot is to create modules that preserve the balance between versatility, low production cost, and functionality. The construction of modules implies that they can be quickly developed and produced at minimum economic costs. The system architecture is formed of modules such as Modules, M-Robots, and Colonies.

Modules are the basic components of the system and can be divided into three main types: power/control modules (P/C-modules), joint modules (J), and specialized modules (S). The M-Robot is an autonomous system that is based on at least one P/C-module and one or more $\mathrm{J}$ and $\mathrm{S}$ modules. The Colony contains several M-Robots operating jointly to accomplish a given task.

The $P / C$-module (power/control) controls over all actuators of other modules, processes the sensor data, and controls the information exchange between other $\mathrm{P} / \mathrm{C}$-modules and the control station. The module contains basic electronic devices, power supply, peripheral communications (CAN-bus and Bluetooth), and connection interfaces.

The module control system is based on the master-slave architecture. Accordingly, there are two software types: master software $(M s)$ and slave software $(S s)$. Ms controls the external communication via Bluetooth and synchronizes $S s$ within the robot configuration. In the $M$-Robot, $M s$ is responsible for preprocessing of instructions and their distribution among the remaining $S s$ via the CAN-bus. The data transmitted within the M-Robot has the form of low-level instructions, such as indicators for feedback control by position, data for synchronization, and data received from sensors and actuators. Ss receives messages via the CAN-bus and executes a desired low-level command.

The Joint module $(J)$ is any active or passive actuator of the rotary, linear (prismatic), or spherical types, and their combinations. The module is controlled by a $P / C$-module, which also supplies it with power. The $J$-module may contain control electronics as well.

The specialized module $(S)$ is designed for executing specific tasks. The module can be a special sensor that conducts data processing of its own, a specialized tool, or inexpensive constructs of passive "extremities" such as foot, wheel, a hybrid of foot and wheel, flange, and platform as an operating body of the robot.

The control over the configuration of a robot or robotic system (Colonies) is implemented through the following two types of communication between modules: Intra-robot and Inter-robot. The Intra-robot communication contains those P/C-modules that are incorporated into the structure of one and the same M-Robot. The Inter-robot connects the M-robot with the control station. The two communication types operate via the CAN-bus and Bluetooth technology, respectively.

The set of modules makes it possible to create different variants of robotic devices. A common variant of the M-Robot is an autonomous device consisting of at least one P/C-module, J-module, and S-module. Different module combinations allow the device to be reconfigured or self-reconfigured.

One of the most important features of the SMART robot is that each module can be designed, on the one hand, as a structurally and constructively simple and inexpensive device and, on the other hand, as a complex and high-performance device with its intellectual component.

The most interesting and promising solution seems to be the SMART robot. However, the use of CAN-bus and Bluetooth as a communication environment restricts the functional capabilities of this robot due to the limited capacity of these communication channels and rules out the possibility of remote and supervisory control over the robot at large distances. In addition, the structure of the information-measuring and control system of MR and the module interaction interfaces do not allow third-party products to be used as modules, which reduces the efficiency of the production of these robots.

The analysis of these examples of different modular heterogeneous robotic systems allows us to conclude that the modular principle of robots construction is of current interest and promising. 


\section{Objectives and tasks}

This study is proposed to be based on the functional-modular principle of the organization of the informationmeasuring and control system (IMCS) of mobile robots (MR). This is a difficult task because it is necessary to identify the functionally complete parts of the IMCS (modules) and develop universal software and hardware interfaces that allow the modules to be combined into systems of various functional purposes. Then, a new feature appears: depending on the MR purpose, its configuration is changed through an appropriate composition of different modules that are independent functional elements.

The system mobility complicates the solution of this problem since the communication of the mobile part with a control system that is located far away (for variants of remote and supervisory control) is based on a radio channel with all of its shortcomings.

To solve this problem, we propose a new approach: consider the IMCS of the robotic system as a local area network (LAN) with mobile nodes and develop appropriate network software that makes the system scalable and reconfigurable [11]. Each LAN node is a modular mobile robot with an IMCS that is also a LAN, the nodes of which are functional modules. The final modules produced on the basis of this principle can be directly used in other robotic systems.

The aim of this study is to develop a functional-modular principle of the construction of the software and hardware unit of heterogeneous mobile robotic systems that are composed of functionally independent modules.

The tasks of this study are:

- Develop a MR IMCS structure allowing the robot to be represented as an interaction of individual functionally complete units - modules.

- Determine the minimum set of functionally complete units of the MR IMCS in terms of their implementation as individual mechatronic and/or electronic modules.

- Formulate the functionality of each module and specify the requirements for the module construction as well as communication channels and interfaces (both hardware and software).

- Develop a universal program interface for the interaction of modules, taking into account their upgradability (without changing the functionality) and the possibility of remote dynamic reprogramming of their computing devices.

- Develop a structure of information interaction between MR modules with scalability, reconfigurability, and hardware implementation.

- Develop a user interface for the MR supervisory control system via the Internet and radio channels.

- Develop and produce a laboratory prototype of functional-modular mobile robots, including sensory, execution, control, computing, communication, and program units.

- Conduct an experimental study of the efficiency of the proposed solutions and variants of functional elements.

\section{Main concept of the functional-modular principle}

The project conception suggests the following main principles for the construction of information-measuring and control systems of a modular MR (functional independence of modules is ensured by):

(1) Each module is a functionally complete electronic or mechatronic device.

(2) The robot's IMCS is constructed as a local area network, the nodes of which are modules.

(3) The principle of distributed control is used.

(4) The hardware unit of modules is constructed using the principle of distributed stabilized power supply.

(5) Each module is supplied with appropriate software (driver).

The functional completeness of modules means a structural and functional independence, which makes it possible to use third-party modules identical to replaced modules by their functionality.

The network principle of ICMS construction makes the robot reconfigurable and scalable. The use of the Ethernet technology provides a capacity of inter-module communication channels of up to $1000 \mathrm{Mbit} / \mathrm{s}$ and makes it possible to use the entire family of TCP/IP protocols and other protocols that are widely used on the Internet together with appropriate libraries. In addition, one can use secure digital radio channels (such as those based on the Wi-Fi standard) for remote control.

The principle of distributed control means the following. Each mechatronic or electronic module has its own information-measuring and control system implemented on the basis of a microcontroller and, when necessary, using its own sensory system and actuators. These elements of the module control system ensure its functional completeness. In this case, each module can independently perform a limited set of functions, and the external (general) control system generates a local purpose, determines the sequence of executable functions, and transmits only the necessary instruction parameters to the module, receiving a report on their execution in response: this generates a data loopback [12]. As a result, the whole computing process implementing the general operation of MR can be parallelized.

The principle of distributed stabilized power supply is implemented as follows. The total power of the robot, including the electric actuator, is supplied by a separate module that contains accumulator batteries. The supply voltage of all electrical components, including microcontrollers, is unstable due to load change. Therefore, each module is provided with its own stable voltage supply (SVS), intended for supply only intramodular devices such as microcontrollers. The 
SVS input receives voltage directly from accumulator storage batteries. When there are input voltage pulsations caused, for example, by starter currents of electric motors, the SVS prevents the possible interruption of the program code by intramodule computing devices. Besides, this distributed power supply does not impose a limit on the power consumption by each module; it is merely necessary to limit the total power consumed by all modules from accumulator storage batteries. If the total power consumption is exceed, one only needs to replace a single module by a module with more capacious batteries. In addition, the released heat is distributed between modules, rather than concentrated in a single power supply unit, which would require radiators.

"The concept of drivers" is that for each mechatronic device a unified network control protocol is created on the basis of existing low-level program interfaces of communication [13]. Then, the producer can relatively easily integrate its device into the IMCS of the modular MR, creating only an additional driver that implements the protocol-based program control instructions and the networking protocols. When a new mechatronic device appears in the network, the software of the central control system automatically puts this device into the network during the interaction with the driver. The fact that each module has a computing device and the network principle of the construction of the IMCS of modular MR make it possible to create an appropriate driver for a given module and thus ensure that third-party modules can be involved in the network without reworking their low-level code.

The functional-modular principle of the construction of mobile robotic systems developed in this project makes it possible to create universal modules, which can be used to construct mobile robotic systems for different purposes [14]. Figure 2 shows possible variants of a heterogeneous modular MR. The wheel robot of vertical design (Fig.2a) can be transformed with the help of a simple modernization into a robot of horizontal design (Fig.2c) or into a raised passableness track-mounted robot (Fig.2b) that is structurally assembled from the same modules.

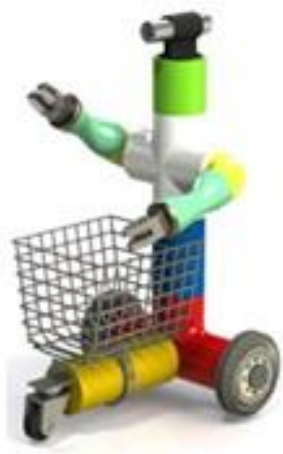

a)

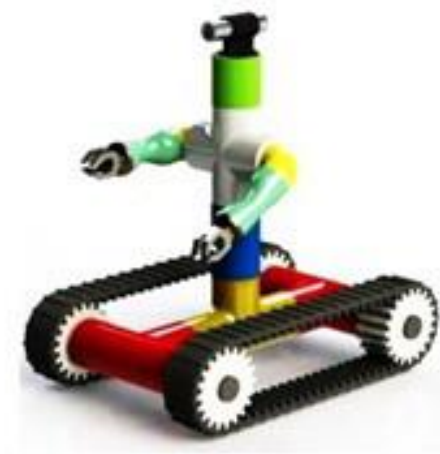

b)

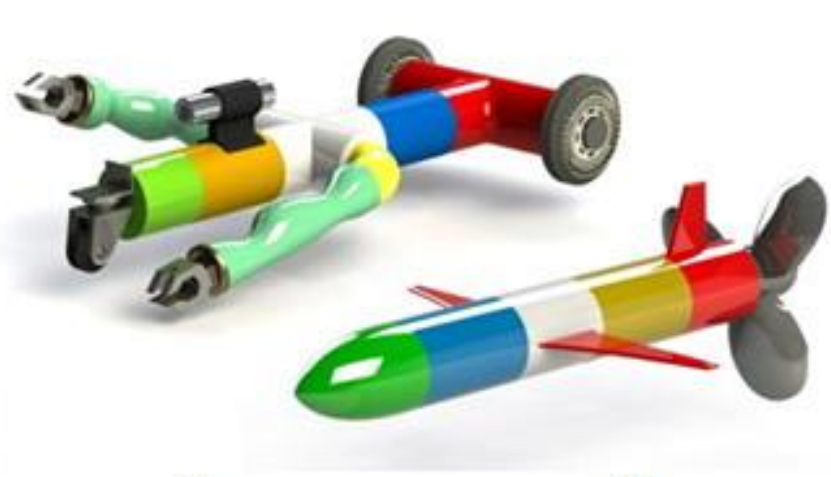

c) d)

Fig. 2. Variants of heterogeneous modular mobile robots (computer prototypes)

Using functionally similar modules but with another design, one can obtain a variant of the underwater research robot (Fig.2d), but the bodies of its modules are made of a more durable material that can withstand high pressure at large depths.

\section{Conclusions}

One of the major challenges of modular robotics is to build a inter-module communication interface. It was necessary to offer a solution to this problem, which would provide a relatively simple integration of ICMS of the individual modules into a single ICMS of the robot. Since the structure of a modular MR is formed from the set of special modules, the type, function, and number of which depend on the robot purpose, its structure should be easily changeable and be made up of modules of various designs and different manufacturers. Mentioned problem is solved by constructing ICMS of the modular robot in the form of local area network in which each module represents a node of the LAN. The simplicity of integration ICMS of modules in ICMS of modular robot is ensured by special software - drivers. Manufacturers of modules should develop the drivers based on the rule set using standard network protocols.

Using this approach, we plan to develop a language of inter-module communication and create a modular service mobile robot equipped with a developed sensor system, two hands-manipulators, and a main computer (Fig.2a). The robot is supposed to have a two-tier (short- and long-range) sensor system. The short-range sensor system supplies the motion module with current data on the surrounding space to ensure that the motion module is a fully functional mechatronic system capable of forming the motion trajectory within the analyzed zone and reacting independently to the emergence of dynamic objects in the zone. This module must make self-decisions on the change of motion trajectory or stopping. This main computer uses the long-range sensor system to build the map of the local area, generate local targets for modules, and develop a general strategy of behavior on the basis of given orders.

The proposed principle of modular design of robotic systems can be successfully used in the Bionic Assembly System $[15,16]$. 


\section{Acknowledgments}

This work was supported by the Russian Foundation for Basic Research, project no. 16-07-00811a.

\section{References}

[1] A.V.Lopota, E.I.Yurevich (2013), Stages and Developments of Robotic Systems Design Modular Principle, In St. Petersburg State Polytechnical University Journal, 1(164), St. Petersburg (Russia). - pp. 98-103

[2] Fukuda T., S. Nakagawa (1987), A dynamically reconfigurable robotic system (concept of a system and optimal configurations), In Proceedings of IECON '87: 1987 International Conference on Industrial Electronics, Control, and Instrumentation

[3] S. Murata, E. Yoshida, A. Kamimura, H. Kurokawa, K. Tomita, and S. Kokaji (2002), M-TRAN: self-reconfigurable modular robotic system, IEEE/ASME Transactions on Mechatronics, 7(4):432-441

[4] E.H. Østergaard, K. Kassow, R. Beck, and H.H. Lund (2006), Autonomous Robots, Design of the ATRON latticebased self-reconfigurable robot, 21(2):165-183

[5] Salemi B., M. Moll, and W.-M. Shen (2006), SUPERBOT: A deployable, multi-functional, and modular selfreconfigurable robotic system, In Proceedings, IEEE/RSJ Intl. Conf. on Intelligent Robots and Systems, pages 36363641, Beijing, China

[6] Fitch R.C. (2004), Heterogeneous Self-Reconfiguring Robotics, PhD thesis, Dartmouth College, Hanover, New Hampshire, USA

[7] Lyder A.H., Stoy K., Mendoza-Garcia R.-F., Larsen J.C., and Hermansen P. (2013), On sub-modularization and morphological heterogeneity in modular robotics, In Sukhan Lee, Hyungsuck Cho, Kwang-Joon Yoon, and Jangmyung Lee (Eds.), Intelligent Autonomous Systems 12, volume 193 of Advances in Intelligent Systems and Computing, pp. 649-661. Springer Berlin Heidelberg

[8] A. Lyder, R.F.M. Garcia, and K. Stoy (2008), Mechanical design of ODIN, an extendable heterogeneous deformable modular robots, In Proceedings, IEEE/RSJ international conference on intelligent robots and systems, ph.883-888, Nice, France

[9] Hancher M. D., Hornby G. S. (2006), A modular robotic system with applications to space exploration, 2nd IEEE International Conference on Space Mission Challenges for Information Technology (SMC-IT'06), Pasadena, CA: IEEE, pp. 132-140

[10] J. Baca, M. Ferre, and R. Aracil (2012), A heterogeneous modular robotic design for fast response to a diversity of tasks, Robotics and Autonomous Systems, vol. 60, no. 4, pp. 522-531

[11] Pryanichnikov V.E., Andreev V.P. (2012), The Application of Network Technologies to Constructing Group Controlled Systems with Machine Vision for Mobile Robots, In Annals of DAAAM for 2012\& Proceedings of the 23th international DAAAM Symposium "Intelligent Manufacturing \& Automation" 24-27th October 2012 Zadar, Croatia, ISSN 2304-1382, V.23, No.1. - pp.1167 - 1174

[12] V.Andreev, K.Kirsanov, and V.Pryanichnikov (2016), Geographically distributed "multi-operator" Control for Mechatronic Devices via the Internet, In Proceedings of the 26th DAAAM International Symposium., B. Katalinic (Ed.), Published by DAAAM International, ISBN: 978-1-5108-1839-2, ISSN 1726-9679, Vienna, Austria. 2015. Curran Proceedings, New York,.pp. 0004-0011

[13] Andreev V.P., Kirsanov K.B., Poduraev Yu. V. (2015), Geographically distributed "multi-operator" control of robotized systems using network technologies, In Proceedings of the International Scientific and Technical Conference "Extreme Robotics". - St.Petersburg: "Politechnika-service", pp. 67-71.

[14] Andreev V., and Kim V., Control System and Design of the Motion Module of a Heterogeneous Modular Mobile Robot, Proceedings of the 27th DAAAM International Symposium, pp.xxxx-xxxx, B. Katalinic (Ed.), Published by DAAAM International, ISBN 978-3-902734-08-2, ISSN 1726-9679, Vienna, Austria DOI: 10.2507/27th.daaam.proceedings.XXX

[15] Katalinic B., Kukushkin I., Pryanichnikov V., Haskovic D. (2014), Cloud Communication Concept for Bionic Assembly System. Procedia Engineering, 1877-7058, 69 (2014) pp. 1562-1568 doi:10.1016/j.proeng.2014.03.156.

[16] Katalinic B., Kukushkin I., \& Haskovic D. (2014), Bionic Assembly System Cloud: Functions, Information Flow and Behavior. In 9th International Conference of DAAAM Baltic, Industrial Engineering, ISBN 978-9949-23-6206, ISSN 2346-6138. pp. 103-108, ed. T.Otto, Tallinn, Estonia 